\title{
Doubling the ultra-wide frequency sweep of linearly chirped lasers by four-wave mixing in a quasi-phase matched nonlinear fiber
}

\author{
Jacob Sendowski, Naresh Satyan, and Amnon Yariv \\ Department of Electrical Engineering, California Institute of Technology, Pasadena, CA, 91125 USA \\ jacobsen@caltech.edu
}

\begin{abstract}
We present an experimental demonstration of the efficient doubling of a wideband $(500 \mathrm{GHz})$ linearly chirped optical waveform by of four wave mixing in a quasi-phase matched nonlinear optical fiber.
\end{abstract}

(C) 2011 Optical Society of America

OCIS codes: $190.4380,190.4370,140.3518$.

Linear swept frequency laser sources have applications in three dimensional imaging and ranging, spectroscopy and sensing. The total range $B(\mathrm{~Hz})$ (bandwidth) of the frequency sweep is the key parameter in many of these applications. In linear chirp ranging and imaging, the axial resolution $z$ is related to the $B$ by $z=c / 2 B$ [1]. One way to multiply the chirp range of a given linear swept frequency laser is via the third order nonlinear process of four wave mixing (FWM).

Previously, using $100 \mathrm{GHz}$ highly linear chirps generated by a semiconductor laser in an optoelectronic phase-lock loop [2], we have demonstrated chirp doubling by four-wave mixing in a $100 \mathrm{~m}$ dispersion flattened highly nonlinear fiber (NLF) [3]. This technique proved effective in generating $200 \mathrm{GHz}$ linear chirps using a simple scheme employing a modest erbium doped fiber amplifier (EDFA). Similar work, extending the tuning range of a mechanically tunable pump laser has also been demonstrated [4]. However, the fiber dispersion and the resulting phase mismatch prevented the efficient doubling of linear chirps with larger frequency sweeps. In this submission, we demonstrate a method for doubling of large bandwidth $(500 \mathrm{GHz})$ linear chirps using a quasi-phase matched (QPM) NLF based on dispersion compensation.

The FWM product output power $P_{\text {out }}$ from a given segment of NLF with a single dispersion parameter is given by:

$$
P_{\text {out }}(L)={ }^{2} P_{\text {ch }}^{2} P_{R} e^{-L}\left(\frac{1-e^{-L}}{2}\right)^{2} \frac{2}{2+2}\left(1+\frac{4 e^{-L} \sin ^{2} \frac{L}{2}}{\left(1-e^{-L}\right)^{2}}\right)
$$

where is the loss per unit length, is the nonlinear gain parameter of the fiber, and $P_{c h}$ and $P_{R}$ are the input powers of the chirped and CW reference waves respectively. The phase mismatch $=2{ }_{c h}-{ }_{R}-{ }_{\text {out }}$ to lowest order in

is $\quad=-2()^{2}$, where is the frequency difference between the pump and signal waves.

From eq. 1 we can see that in the case of low propagation loss $(<<1), P_{\text {out }}$ varies as the square of the length of the nonlinear fiber. However, the quantity $(L)$, which increases as the square of the frequency difference between the chirp and reference waves, limits the conversion efficiency bandwidth of this process. For given input powers $P_{R}$ and $P_{c h}$, this represents an undesirable trade off between the interaction length and conversion efficiency bandwidth for the FWM process.

Quasi phase matching via dispersion compensation is a known method for overcoming phase matching limitations arising in nonlinear optics [5]. By cascading multiple short segments of NLF while periodically alternating the sign of the dispersion parameter $D$ in each segment, it is possible to engineer a nonlinear medium that has a long interaction length while also achieving a large conversion efficiency bandwidth.

We demonstrated frequency sweep doubling using four $50 \mathrm{~m}$ segmetns of nonlinear fiber (OFS Fitel zero slope NLF). The group velocity dispersion parameters of the fibers were $-0.59,+0.38,-0.59,+0.38$ in units of ps/nm$\mathrm{km}$. The measured conversion efficiency was found to agree well with theory as shown in Fig. 2 (a) This arrangement exhibited a $3 \mathrm{~dB}$ conversion efficiency bandwidth of approximately $1.5 \mathrm{THz}$, nearly $700 \mathrm{GHz}$ more than the theoretical $3 \mathrm{~dB}$ conversion efficiency bandwidth of a $200 \mathrm{~m}$ single segment of HNLF with a dispersion parameter $D=+0.38 \mathrm{ps} / \mathrm{nm}-\mathrm{km}$. 


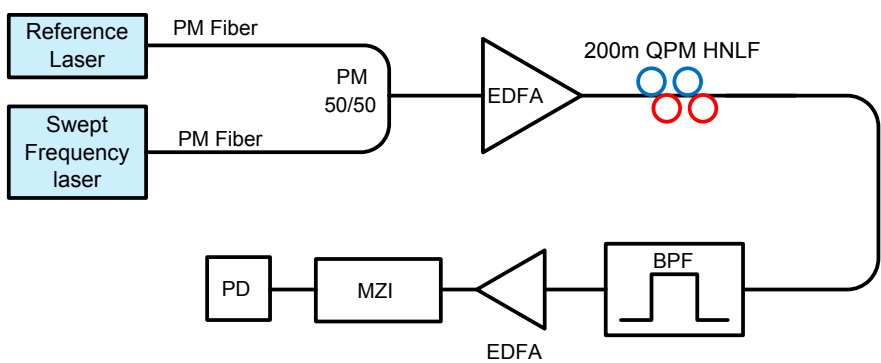

Fig. 1. Schematic diagram of the experimental setup for chirp bandwidth doubling in QPM NLF. EDFA: Erbium doped fiber amplifier, BPF: Band pass filter, MZI: Mach-Zehnder interferometer, PD: Photodetector

We also performed a chirp doubling experiment using a $500 \mathrm{GHz}$ in $400 s$ linear chirp signal from an optoelectronic swept frequency laser similar to that of [2]. The chirp input was mixed with a CW reference beam as shown in Fig. 1. We obtained a $1 \mathrm{THz}$ in $400 s$ linear chirp. These results are shown in Fig. 2. The signal to noise ratio of the output chirp is roughly $25 \mathrm{~dB}$, a decrease from that of the input chirp due mostly to the amplified spontaneous emission (ASE) noise of the EDFA as seen in the optical spectrum of Fig. 2(b). The phase noise of the SFL is also doubled by the FWM process and contributes to the degraded SNR as well.

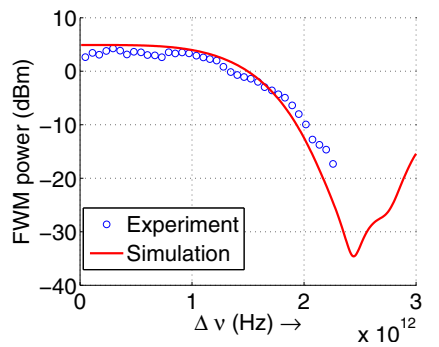

(a)

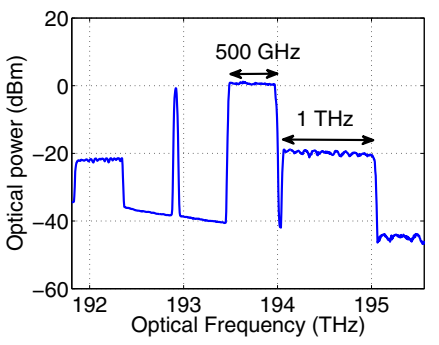

(b)

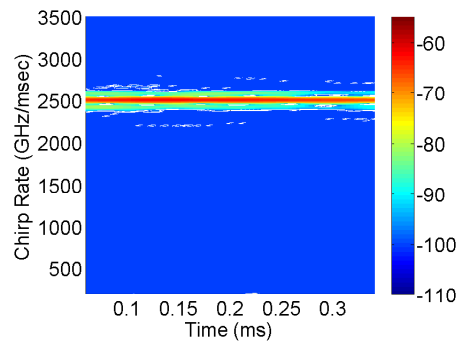

(c)

Fig. 2. (a) Experimental demonstration of chirp doubling in a four segment QPM HNLF, (b) the spectrogram of photocurrent of the filtered FWM output, and (c) the conversion efficiency spectrum of the $200 \mathrm{~m}$ QPM HNLF used in the experiments. $P_{c h}=100 \mathrm{~mW}$ and $P_{R}=100 \mathrm{~mW}$.

The extension of this method to a greater number of shorter segments than those used here, will allow for larger conversion efficiency bandwidths while enabling longer interaction lengths and therefore greater nonlinear power output. Multiple stages of chirp multiplication could be used to generate chirps with bandwidths of 5-10 THz corresponding to axial resolutions of 15 to $20 \mathrm{~m}$ relevant for biological imaging.

\section{References}

1. W. Burdic, Radar signal analysis, Prentice-Hall electrical engineering series (Prentice-Hall, 1968).

2. N. Satyan, A. Vasilyev, G. Rakuljic, V. Leyva, and A. Yariv, "Precise control of broadband frequency chirps using optoelectronic feedback," Opt. Express 17, 15,991-15,999 (2009).

3. N. Satyan, G. Rakuljic, and A. Yariv, "Chirp multiplication by four wave mixing for wideband swept-frequency sources for high resolution imaging," J. Lightwave Technol. 28, 2077-2083 (2010).

4. B. P.-P. Kuo and S. Radic, "Fast wideband source tuning by extra-cavity parametric process," Opt. Express 18, 19,930-19,940 (2010).

5. K. Inoue, "Arrangement of fiber pieces for a wide wavelength conversion range by fiber four-wave mixing," Opt. Lett. 19, 1189-1191 (1994). 